\title{
A 2D CHAOS-BASED VISUAL ENCRYPTION SCHEME FOR CLINICAL EEG SIGNALS
}

Chin-Feng Lin

Department of Electrical Engineering, National Taiwan Ocean University, Keelung, Taiwan, R.O.C., Icf1024@mail.ntou.edu.tw

Boa-Shun Wang

Department of Electrical Engineering, National Taiwan Ocean University, Keelung, Taiwan, R.O.C.

Follow this and additional works at: https://jmstt.ntou.edu.tw/journal

Part of the Engineering Commons

\section{Recommended Citation}

Lin, Chin-Feng and Wang, Boa-Shun (2011) "A 2D CHAOS-BASED VISUAL ENCRYPTION SCHEME FOR CLINICAL EEG SIGNALS," Journal of Marine Science and Technology. Vol. 19: Iss. 6, Article 11.

DOI: $10.51400 / 2709-6998.2209$

Available at: https://jmstt.ntou.edu.tw/journal/vol19/iss6/11

This Research Article is brought to you for free and open access by Journal of Marine Science and Technology. It has been accepted for inclusion in Journal of Marine Science and Technology by an authorized editor of Journal of Marine Science and Technology. 


\section{A 2D CHAOS-BASED VISUAL ENCRYPTION SCHEME FOR CLINICAL EEG}

SIGNALS

\section{Acknowledgements}

The authors acknowledge the support of the teacher research project of National Taiwan Ocean

University, 99B60201, Center for Marine Bioenvironment and Biotechnology, NSC 93-2218-E-019-024, and the valuable comments of the reviewers. 


\title{
A 2D CHAOS-BASED VISUAL ENCRYPTION SCHEME FOR CLINICAL EEG SIGNALS
}

\author{
Chin-Feng Lin* and Boa-Shun Wang*
}

Key words: two dimensional (2D) chaos-based encryption scheme, clinical electroencephalography, transmission bit error rate, parallel processing, mobile telemedicine.

\begin{abstract}
In this paper, we have developed a two-dimensional (2D) chaos-based encryption scheme that can be applied to signals with transmission bit errors in clinical electroencephalography (EEG) and mobile telemedicine. As opposed to one-dimensional (1D) chaos-based encryption, the proposed 2D schemes uses the concept of parallel processing to increase the encryption speed. An essential feature of the proposed scheme is that signals mapping of a $2 \mathrm{D}$ chaotic scrambler and a permutation scheme are used to obtain clinical EEG information that requires high-level encryption. Simulation results show that when the correct deciphering parameters are inputted, EEG signals with a transmission bit error rate (BER) of $10^{-7}$ are completely recovered. However, these signals can not be recovered if there is an error in the input parameters, for example, an initial point error of $0.00000001 \%$.
\end{abstract}

\section{INTRODUCTION}

Chaos theory is an interesting branch of mathematics that has been widely applied in many engineering fields [2-31]. One application of this theory is chaos-based encryption, which is used for the encryption of data, audio, video, images, and biomedical signals. Unlike block encryption algorithms such as the data encryption standard (DES) algorithm, Rivest, Shamir, and Adleman (RSA) algorithm, and advanced encryption standard (AES) algorithm, a chaos sequence is continuous and suitable for data encryption in continuous media such as audio, video, electrocardiogram (ECG) and electroencephalography (EEG) signals, and in large block files such as image signals. Chaos-based encryption is sensitive to the starting point and type of the chaotic logistic map used; different starting points and chaotic logistic maps generate different chaotic sequences for encryption. Zhou et al. [31]

Paper submitted 08/12/09; revised 02/23/10, 04/12/10; accepted 06/09/2010. Author for correspondence: Chin-Feng Lin (e-mail: lcf1024@mail.ntou.edu.tw). *Department of Electrical Engineering, National Taiwan Ocean University, Keelung, Taiwan, R.O.C. identified several disadvantages of previous chaos-based encryption system: (i) the chaotic map may be analyzed easily if a few plaintext-ciphertext pairs are known; (ii) the selfsynchronization subsystem is insensitive to the parameters used; and (iii) the signal to be encrypted is strongly correlated to the original signal. In [26], an efficient hierarchical chaotic image encryption algorithm has been developed on the basis of chaotic permutation and used for rearranging image blocks and for scrambling the pixels in each block. In $[2,3,17,20,21$, 24-26], chaos-based encryption schemes that can be applied to e-mail, voice, and video signals have been proposed. In these studies, chaos-based encryption algorithms have been adopted to generate encrypted bit streams. In these encryption algorithms, an exclusive-OR (XOR) gate is used to generate encryption e-mail, voice, and video bit streams. Chaos-based decryption carried out in the receiver by using the same parameters used for chaos-based encryption and decrypted e-mail, voice, and video bit streams are generated by carrying out an XOR operation. Chaos-based encryption is suitable for mobile telemedicine applications $[11-14,16]$, as it guarantees confidentiality of patient-related information by facilitating data protection. In [12], we carry out chaos-based pixel address (position) permutation and transformation of pixel values for the encrypt ion X-ray images. We use all-pass filtering to scramble the phase spectra of the most-important lowresolution sub-image in the following manner. In the pre-filtering step, we add 2D chaotic signals to randomize reference phase spectra; then, in the post-filtering step, we subtract the same reference phase spectra to recover the original phase spectra of the image. In [14], we proposed a one-dimensional (1D) chaos-based bit streams ciphers for use in mobile telemedicine systems. In [15], we scramble the signal values of the inputted EEG signals by a 1D chaotic signal to randomize the EEG signal values, and applied chaotic address scanning order encryption to achieve visual encryption. In this paper, we make improvements to 1D chaos-based encryption and propose a $2 \mathrm{D}$ chaos-based visual encryption scheme based on parallel processing for application to clinical EEG signals. The proposed 2D chaos-based cipher can simultaneously encrypt $N$ clinical channels in a parallel architecture. An essential feature of the 2D encryption scheme is that signal mapping of $2 \mathrm{D}$ chaotic scrambler and a permutation scheme are used to obtain clinical EEG information that requires high-level encryption. The encryption speed in the case of $2 \mathrm{D}$ 


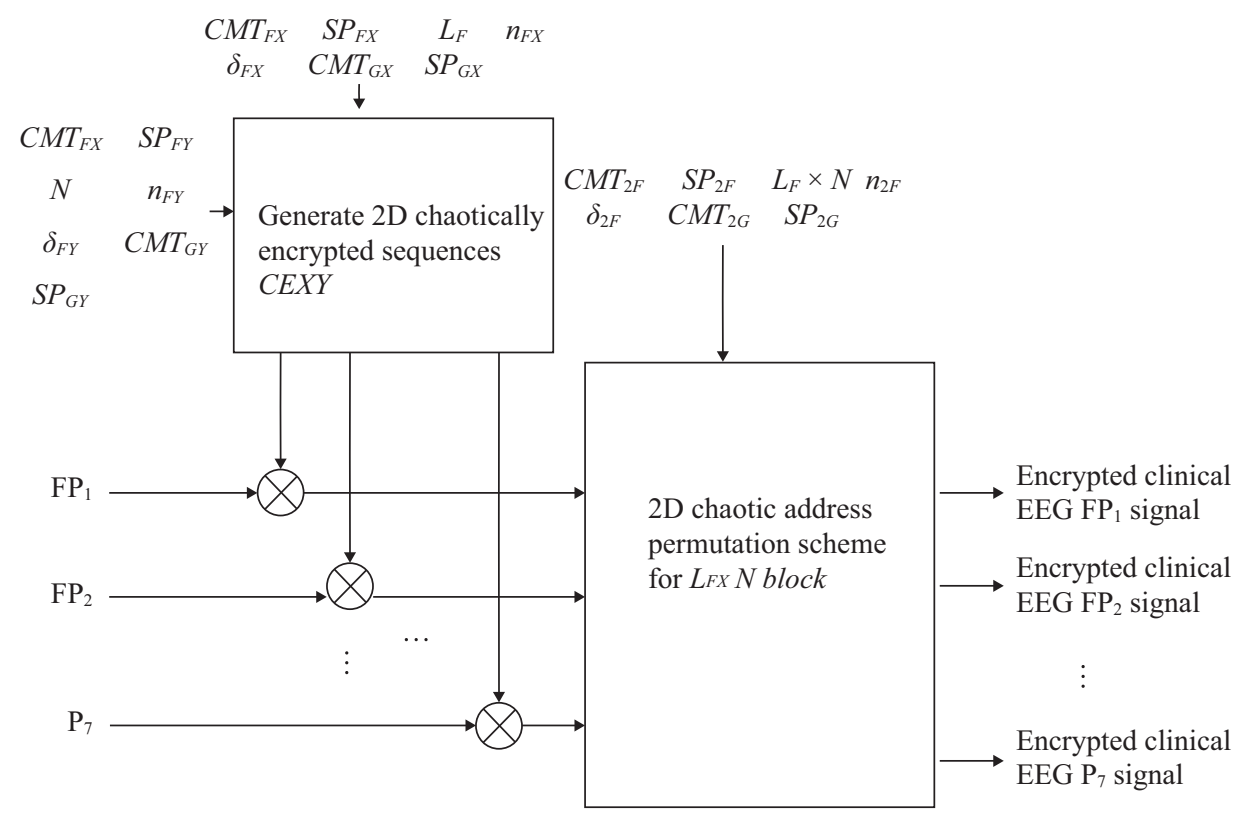

Fig. 1. Proposed 2D chaos-based encryption scheme for clinical EEG signals.

encryption is $N$ times higher than that in the case of $1 \mathrm{D}$ chaos-based encryption; on the other hand, the hardware complexity in 2D encryption is $N$ times lower than that in 1D encryption. This is because part of the hardware can be hardware shared in the proposed 2D chaos-based cipher. Simulation results show that when the correct deciphering parameters are inputted, signals with a transmission bit error rate (BER) of $10^{-7}$ are completely recovered. However, signal recovery is not achieved if there is an error in the input parameters, for example, an input point error of $0.00000001 \%$.

\section{A 2D CHAOS-BASED ENCRYPTION SCHEME FOR CLINICAL EEG SIGNALS}

Fig. 1 shows the architecture of the proposed 2D chaosbased encryption scheme to clinical EEG signals. In this scheme, a 2D chaos-based encryption scrambler and a 2D chaotic address permutation method are used. First, $N$ multichannel clinical EEG signals are inputted to the 2D chaosbased cipher and processed by a 2D chaos-based encryption scrambler to generate $1^{\text {st }} 2 \mathrm{D}$ chaotically encrypted EEG signals. In addition, 2D chaotically encrypted sequences are generated, and are multiplied by the $N$ multichannel clinical EEG signals to obtain the $1^{\text {st }} 2 \mathrm{D}$ chaotically encrypted EEG signals. Then, the $1^{\text {st }} 2 \mathrm{D}$ chaotically encrypted EEG signals are processed by the $2 \mathrm{D}$ chaotic address permutation method to generate a $2^{\text {nd }} 2 \mathrm{D}$ chaotically encrypted EEG signals. Fig. 2 shows the flow chart of the proposed 2D visual chaos-based encryption scheme for clinical EEG signals. In order to increase the robustness of the encryption system, we use chaotic index address assignment process $F_{C I A X}$ and chaotic candidate point generator process $G_{C C S X}$ to represent values in the x coordinate axis, and to generate 1D chaotically encrypted sequences (CEX) of length $L_{F}$. Similarly, the process is repeated to generate $1 \mathrm{D}$ chaotically encrypted sequences $(C E Y)$ of length $N$ in the y coordinate axis. The vector inner product of $C E X$ and $C E Y$ is obtained to generate $2 \mathrm{D}$ chaotically encrypted sequences $C E X Y$. The $1^{\text {st }} 2 \mathrm{D}$ chaotically encrypted signals $C E E G 1$ is generated from the vector inner product of $C E X Y$ and $N$ channel clinical $E E G$ signals. In order to decrease the correlation between the encrypted and original EEG signals, we use $2 \mathrm{D}$ chaotic address permutation encryption to generate $2^{\text {nd }} 2 \mathrm{D}$ chaotically encrypted EEG signals SGEEGN. The parameters used in the scheme are listed in Table 2. The steps involved in the implementation of our 2D chaos-based encryption scrambler can be summarized as shown below.

Step 1: Select a chaotic logistic map type $C M T_{F X}$ of $F_{C I A X}$, where the starting point is $S P_{F X}, L_{F}$ is the length of an encrypted clinical EEG signal. The parameters $n_{F X}$ and, $\delta_{F X}$ denote the security level.

Step 2: Generate a chaotic sequence of length $n_{F X}$.

$$
\begin{aligned}
& x_{n+1}=C M T_{F X}\left(x_{n}\right) ; x_{0}=S P_{F X} \\
& n=\left\{1,2, \ldots, n_{F X}\right\}
\end{aligned}
$$

Step 3: Discard the previous $n_{F X}$ chaotic sequence.

Step 4: Generate a new chaotic sequence

$$
x_{n}=C M T\left(x_{n_{F X}+1}\right) ; n=\left\{n_{F X}+1, \ldots\right\} ;
$$

Step 5: If $x_{n}>\delta_{F X}$, discard $x_{n}$ and go to step 4. Else, go to step 6 . 
Table 1. The performance of transmission error of the proposed $2 \mathrm{D}$ chaos-based clinical EEG signals.

\begin{tabular}{|c|c|c|c|c|c|c|c|c|}
\hline & BER & $10^{-7}$ & $10^{-6}$ & $10^{-5}$ & $10^{-4}$ & $10^{-3}$ & $10^{-2}$ & $10^{-1}$ \\
\hline \multirow{3}{*}{ Correct encryption } & PRD (\%) & 1.1285 & 1.1285 & 1.1744 & 1.2069 & 6.1534 & 20.0253 & 61.2303 \\
\cline { 2 - 9 } & $\mathrm{MSE}$ & 0.0056 & 0.0056 & 0.0061 & 0.0064 & 0.167 & 1.7691 & 16.5397 \\
\cline { 2 - 9 } & $\mathrm{r}$ & 0.9999 & 0.9999 & 0.9999 & 0.9999 & 0.9981 & 0.9804 & 0.8288 \\
\hline Error encryption & $\mathrm{r}$ & 0.0272 & 0.0272 & 0.0272 & 0.0272 & 0.0276 & 0.0297 & 0.0341 \\
\hline
\end{tabular}

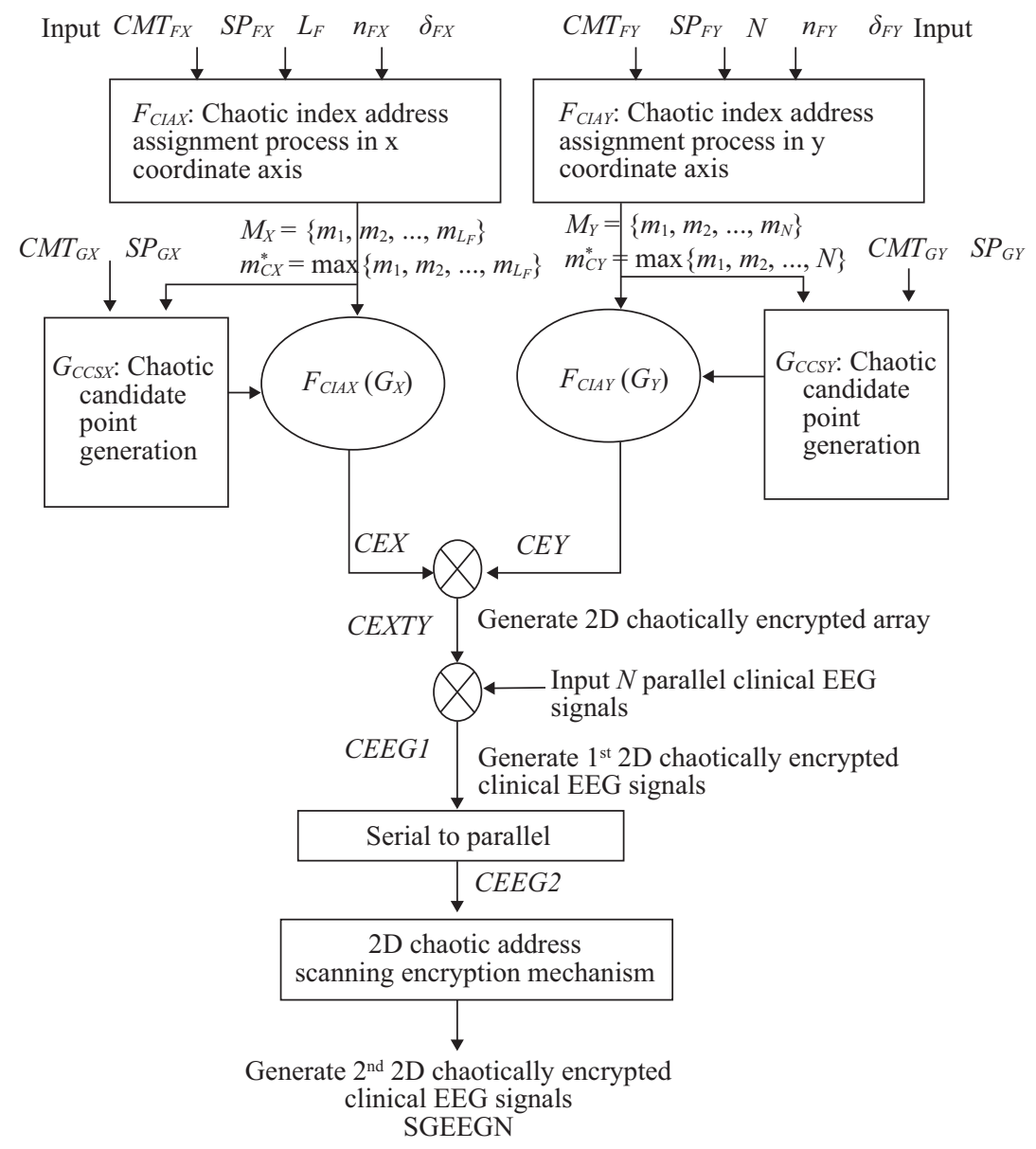

Fig. 2. Flow chart of the proposed 2D visual chaos-based encryption scheme for clinical EEG signals.

Step 6:

$$
m_{k}=\left\lceil\frac{1}{x_{n}}\right\rceil
$$

If $m_{k} \notin M_{X} ; M_{X}=\left\{m_{1}, \ldots, m_{k-1}, m_{k}\right\}$, go to step 7. Else, go to step 4.

Step 7: If $k=L_{F}$;

$$
\begin{aligned}
& M_{X}=\left\{m_{1}, m_{2}, \ldots, m_{L_{F}}\right\} ; \\
& m_{C X}^{*}=\max \left\{m_{1}, m_{2}, \ldots, m_{L_{F}}\right\}
\end{aligned} ;
$$

(3) Step 11: Deliver $M$ to the chaotic candidate point generator coordinate axis.

Else, go to step 4 . $G_{C C S X}$.

Step 12: Generate an encrypted chaotic signal $C E X$ in the $\mathrm{x}$

Step 8: Deliver $m_{C X}^{*}$ to the chaotic candidate point generator $G_{C C S X}$.

Step 9: Deliver a chaotic logistic map of the type $C M T_{G X}$ for $G_{C C S X}$, where the starting point is $S P_{G X}$.

Step 10: Generate a chaotic sequence of length $m_{C}^{*}$.

$$
\begin{aligned}
& g_{n+1}=C M T_{G X}\left(g_{n}\right) ; \\
& g_{0}=S P_{G X} ; n=\left\{1,2, \ldots, m_{C}^{*}\right\} ; \\
& G_{X}=\left\{g_{1}, \ldots, g_{\left.m_{C}^{*}\right\}}\right.
\end{aligned}
$$


$M_{X}=\left\{m_{1}, m_{2}, \ldots, m_{L_{F}}\right\} ; G_{X}=\left\{g_{1}, \ldots, g_{m_{C}^{*}}\right\} ;$

$C E \mathrm{X}=\left\{g_{m_{1}}, g_{m 2}, \ldots, g_{m_{L_{F}}}\right\}=\left\{c \operatorname{cex}_{1}, c e x_{2}, \ldots, c e x_{L_{F}}\right\}$

Step 13: Repeat steps 1-12 to generate an encrypted chaotic signal $C E Y$ in the y coordinate axis. The length of the $C E Y$ signal is $N$.

Step 14: Generate a 2D chaotically encrypted sequence $C E X Y$.

$$
\begin{gathered}
C E \mathrm{X}=\left\{c e x_{1}, c e x_{2}, \ldots, c e x_{L_{F}}\right\} \\
C E \mathrm{Y}=\left\{c e y_{1}, c e y_{2}, \ldots, c e y_{L_{F}}\right\} \\
C E X Y=\left[\begin{array}{ccccc}
c e x_{1} \times c e y_{1} & c e x_{2} \times c e y_{1} & \cdots & c e x_{L_{F X}} \times c e y_{1} \\
c e x_{1} \times c e y_{2} & c e x_{1} \times c e y_{2} & \cdots & c e x_{L_{F X}} \times c e y_{2} \\
\vdots & \vdots & \vdots & \vdots \\
c e x_{1} \times c e y_{N} & c e x_{2} \times c e y_{N} & \cdots & c e x_{L_{F X}} \times c e y_{N}
\end{array}\right] \\
=\left[\begin{array}{cccc}
c e x y_{11} & c e x y_{12} & \cdots & c e x y_{1 L_{F}} \\
c e x y_{21} & c e x y_{22} & \cdots & c e x y_{2 L_{F}} \\
\vdots & \vdots & \vdots & \vdots \\
c \operatorname{exy}_{N 1} & c \operatorname{cxy}_{N 2} & \cdots & c \operatorname{exy}_{N L_{F}}
\end{array}\right]
\end{gathered}
$$

Step 15: Input $N$ parallel clinical EEG signals. The length of the clinical EEG signal sequences is $L_{F}$. The $N$ clinical EEG signals are defined as

$$
E E G=\left[\begin{array}{cccc}
e e g_{11} & e e g_{12} & \cdots & e e g_{1 L_{F}} \\
e e g_{21} & e e g_{22} & \cdots & e e g_{2 L_{F}} \\
\vdots & \vdots & \vdots & \vdots \\
e e g_{N 1} & e e g_{N 2} & \cdots & e e g_{N L_{F}}
\end{array}\right]
$$

Step 16: Generate $1^{\text {st }}$ 2D chaotic clinical EEG signals CEEG1, which are given as

$C E E G 1=E E G \times C E X Y=\left[\begin{array}{cccc}c e e g 1_{11} & c e e g 1_{12} & \cdots & c e e g 1_{1 L_{F}} \\ c e e g 1_{21} & c e e g 1_{22} & \cdots & c e e g 1_{2 L_{F}} \\ \vdots & \vdots & \vdots & \vdots \\ c e e g 1_{N 1} & c e e g 1_{N 2} & \cdots & c e e g 1_{N L_{F}}\end{array}\right]$

Then, input the $N$ parallel $1^{\text {st }} 2 \mathrm{D}$ chaotic clinical EEG Signals $C E E G 1$ to a serial-to-parallel converter and generate an $N \times L_{F}$ chaotic clinical EEG signals $C E E G 2$, which are given as

$$
\begin{aligned}
& C E E G 1=\left\{\begin{array}{llllll}
\operatorname{ceeg} 1_{11} & \operatorname{ceeg} 1_{12} & \cdots & \operatorname{ceeg} 1_{1 L_{F}} & \cdots & \operatorname{ceeg} 1_{N L_{F}}
\end{array}\right\} \\
& \text { CEEG2 }=\left\{\begin{array}{llll}
\operatorname{ceeg} 2_{1} & \operatorname{ceeg} 2_{2} & \cdots & \operatorname{ceeg} 2_{N \times L_{F}}
\end{array}\right\}
\end{aligned}
$$

Process the chaotic clinical EEG signals CEEG2 by using $2 \mathrm{D}$ chaotic address permutation method, and output the $2^{\text {nd }} 2 \mathrm{D}$ chaotically encrypted clinical EEG signals.

The $2 \mathrm{D}$ chaotic address permutation encryption scheme is described as follows.

Steps 1 to 5 are the same as those described for the $1^{\text {st }} 2 \mathrm{D}$ chaos-based encryption scheme.

Step 6:

$$
m_{k I}=\left\lceil\frac{1}{x_{n}}\right\rceil
$$

If $m_{k 1} \leq N \times L_{F} ; m_{k 1} \notin\left\{m_{1}, \ldots, m_{k-1}\right\}$;

$M=\left\{m 1, \ldots, m_{k 1-1}, m_{k 1}\right\}$; go to step 7 .

Else, go to step 4;

Step 7: If $k 1=N \times L_{F}$

$$
M=\left\{m_{1}, m_{2}, \ldots, m_{\mathrm{N} \times L_{F}}\right\} ; \text { else, go to step } 4 .
$$

Step 8: Deliver $M$ to the output encrypted signal processor.

Step 9: Deliver the encrypted clinical EEG signals CEEG2 to output the encrypted signals.

Step 10: Perform chaotic address permutation of the encrypted clinical EEG signal SGEEG.

$$
\begin{aligned}
\text { GEEG } & =\left\{\text { geeg }_{1}, \ldots, \text { geeg }_{\mathrm{N} \times L_{F}}\right\} ; \\
M & =\left\{m_{1}, m_{2}, \ldots, m_{\mathrm{N} \times L_{F}}\right\} \\
\text { SGEEG } & =\left\{\text { geeg }_{m_{1}}, \text { geeg }_{m_{2}} \ldots, \text { geeg }_{m_{\mathrm{N} \times L_{F}}}\right\} \\
& =\left\{\text { seeg }_{1}, \text { seeg }_{2}, \ldots, \text { sgeeg }_{\mathrm{N} \times L_{F}}\right\}
\end{aligned}
$$

Step 11: Input $S G E E G$ to a serial-to-parallel converter, and generate $2^{\text {nd }} 2 \mathrm{D}$ chaotically encrypted clinical EEG signals SGEEGN.

$$
\text { SGEEGN }=\left[\begin{array}{cccc}
\text { sgeeg }_{1} & \text { sgeeg }_{2} & \cdots & \text { sgeeg }_{L_{F}} \\
\text { sgeeg }_{L_{F+1}} & \text { sgeeg }_{L_{F}+2} & \cdots & \text { sgeeg }_{2 L_{F}} \\
\vdots & \vdots & \vdots & \vdots \\
\text { sgeeg }_{(N-1) \times L_{F}+1} & \text { sgeeg }_{(N-1) \times L_{F}+2} & \cdots & \text { sgeeg }_{N \times L_{F}}
\end{array}\right]
$$

If the abovementioned process is carried out in the reverse order, the $N$ parallel clinical EEG signals are decrypted.

\section{SIMULATION RESULTS}

Fig. 3 shows the eight parallel clinical EEG signals FP1, FP2, ..., P8, P7 in the EEG database [5]. The sampling rate of each clinical signal channel is 256 samples/s. The following are the parameters used in the proposed 2D chaos-based encryption scheme. The starting points are $S P_{G X}=0.100011$, and $S P_{F X}=0.200011, m_{C}^{*}=256, N=8, n_{F X}=n_{F Y}=25600$, and 


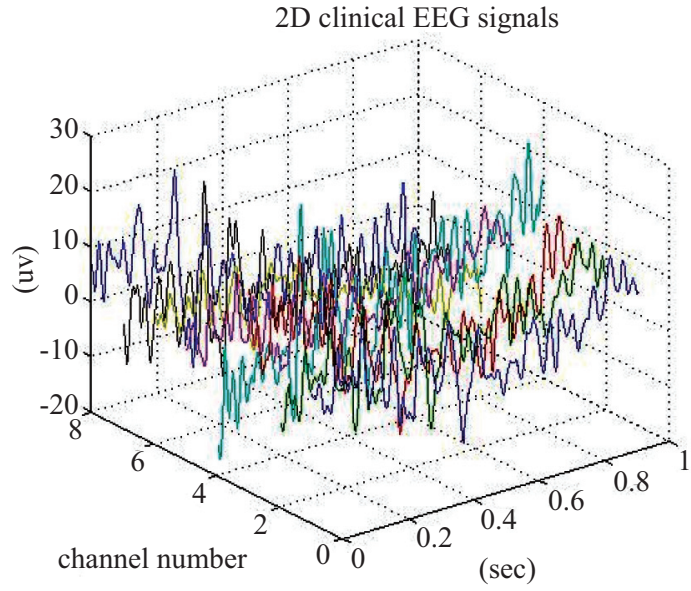

Fig. 3. Eight parallel clinical EEG signals.

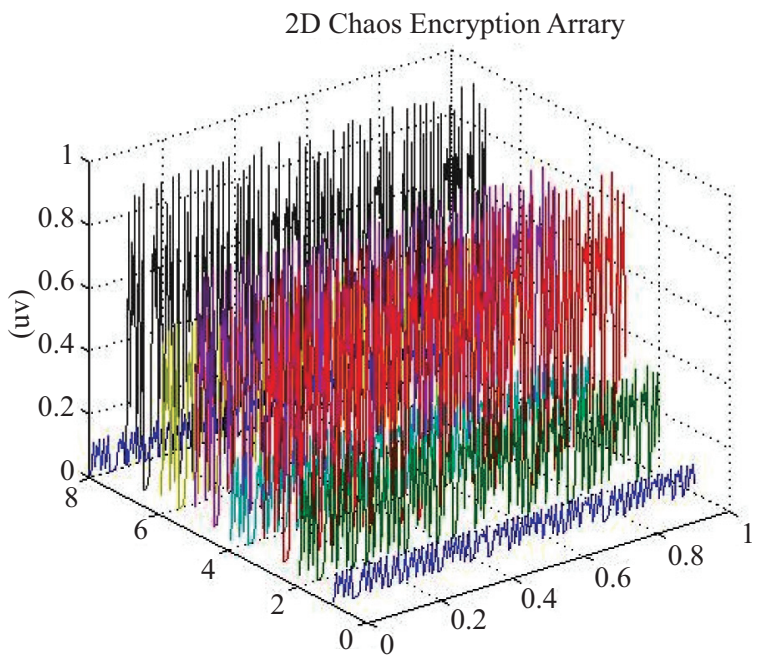

Fig. 4. 2D chaotically encrypted array.

$\delta_{F X}=\delta_{F Y}=0.1$. The chaotic equation $x_{n+1}=r x_{n}\left(1-x_{n}\right)$ is applied to all types of chaotic logistic maps. Figs. 4 and 5 show the 2D chaotically encrypted array and the 2D chaotically encrypted EEG signals, respectively. We discuss the difference between the original and chaotically encrypted EEG signals by using the Pearson correlation coefficient $\gamma$, which is given by

$$
r=\frac{\sum X Y-\frac{\sum X \sum Y}{Z}}{\sqrt{\left(\sum X^{2}-\frac{\left(\sum X\right)^{2}}{Z}\right)\left(\sum Y^{2}-\frac{\left(\sum Y\right)^{2}}{Z}\right)}}
$$

Here, $X$ and $Y$ are the amplitudes of the original and encrypted EEG signals, respectively. $Z$ is the total number of sampled EEG signals. The $r$ value of signals $\mathrm{A}$ and $\mathrm{B}$ is 1 , which indicates that these signals $\mathrm{A}$ and $\mathrm{B}$ are identical and completely correlated. The $r$ value is 0.11 for the original clinical EEG signals and the chaotically encrypted signal

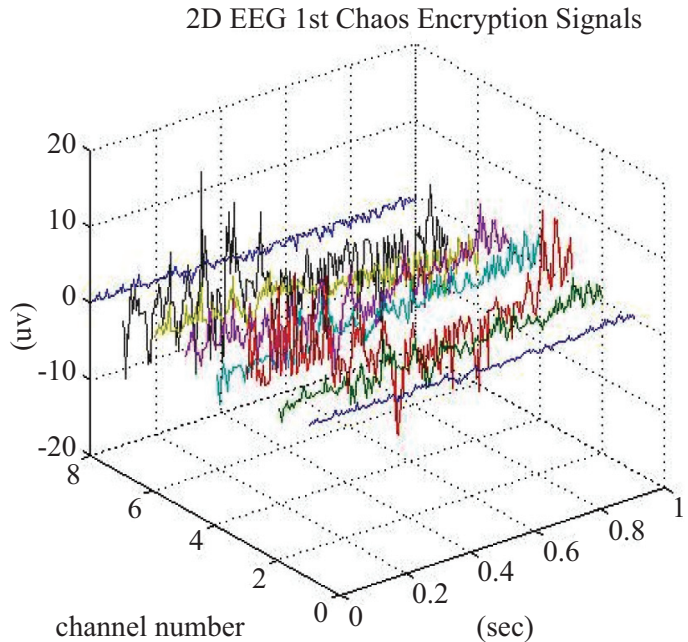

Fig. 5. The $1^{\text {st }} 2 \mathrm{D}$ chaotically encrypted clinical EEG signals generated by using the proposed chaotic scrambler. $(r=0.11)$

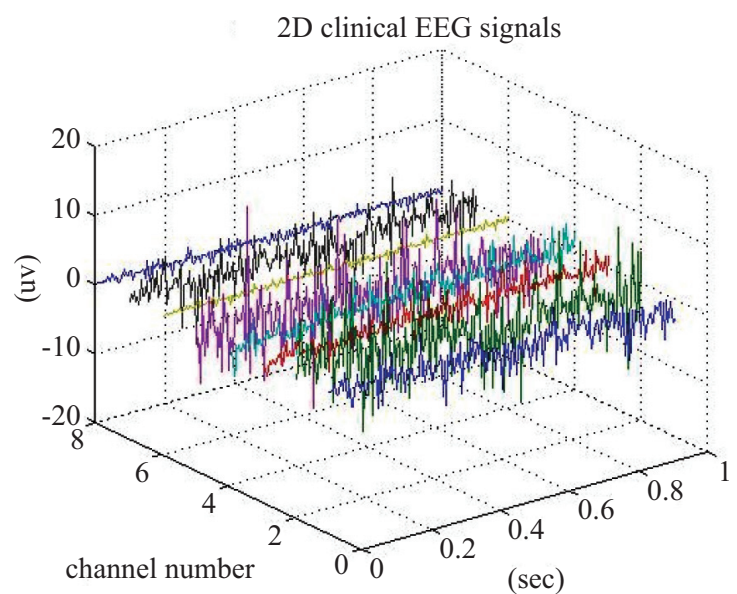

Fig. 6. The $2^{\text {nd }} 2 \mathrm{D}$ chaotically encrypted clinical EEG signals generated by using the proposed chaotic scrambler and $2 \mathrm{D}$ chaotic address permutation method. $(r=0.016)$

(transmission BER of $10^{-7}$ ) generated by using the proposed chaos-based encryption scrambler. Fig. 6 shows the 2D EEG signals that are chaotically encrypted by the proposed encryption scrambler and the $2 \mathrm{D}$ chaotic address permutation method. The transmission BER is $10^{-7}$. The $r$ value of the original clinical EEG signals and those encrypted using the proposed encryption scheme is 0.0272 . In this case, the encrypted clinical EEG signal are unreadable. The percent root-mean-square difference $(P R D)$ is used to evaluate the distortion of the decrypted signals. The $P R D$ value [1] is obtained using the equation

$$
P R D=100 \times \sqrt{\frac{\sum_{i=1}^{L}\left(X_{o r i}(i)-X_{d e c}(i)\right)^{2}}{\sum_{i=1}^{L} X_{\text {ori }}^{2}(i)}}
$$




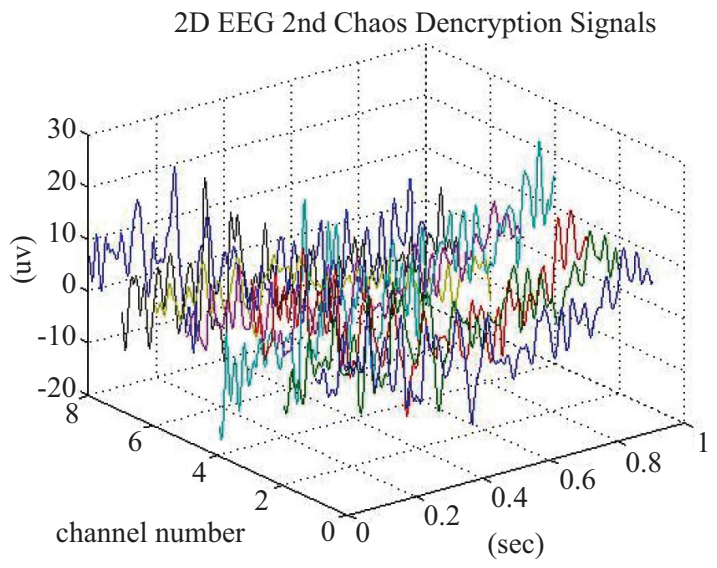

Fig. 7. 2D EEG chaos decrypted signals. $\left(B E R=10^{-7}\right.$ and $\left.P R D=1.1285 \%\right)$.

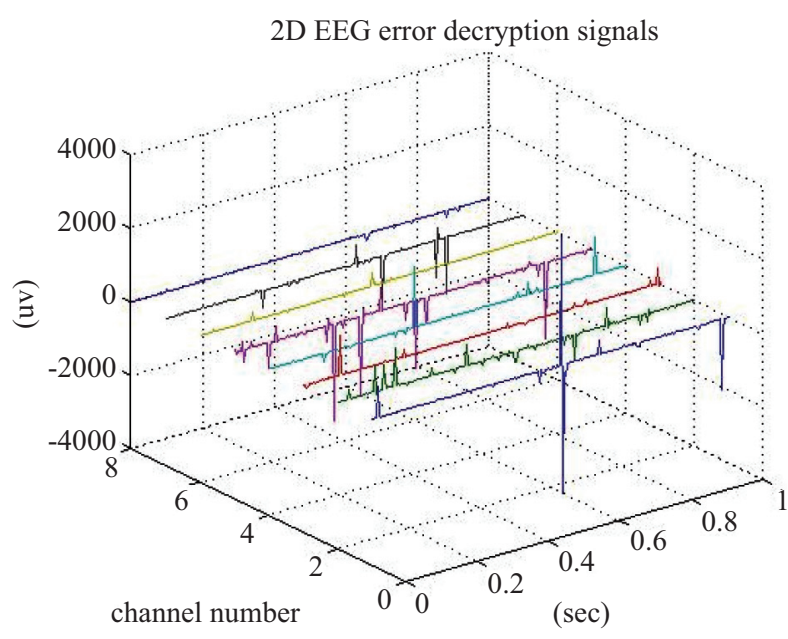

Fig. 8. 2D decrypted EEG signals with error decryption parameter.

where $X_{o r i}$ and $X_{d e c}$ are the original and decrypted clinical EEG signals, respectively. The correct decrypted clinical EEG signals are shown in Fig. 7. We assume that the received EEG signals with a transmission BER of $10^{-7}$. The PRD value of the correct decrypted EEG signals and the original EEG signals is $1.1285 \%$. Fig. 8 shows that the error in the decrypted EEG signals and the decrypted parameter at the starting point is $10^{-6}$. From the values shown in Table 1, we can discuss the effect of the transmission BERs in the EEG signals that are encrypted by using the proposed scheme. The $P R D$ and $r$ values of the correct encrypted signals are the same for BER $=10^{-7}$ and $10^{-6}$. The decrypted EEG signals are distorted when the transmission BER exceeds $10^{-3}$. From theses simulation results, we conclude that the proposed chaos-based $2 \mathrm{D}$ encryption is a superior scheme that is well suited for application to clinical EEG signals.

\section{CONCLUSION}

In this paper, we have developed a high-speed encryption scheme based on the chaos theory for application to clinical
Table 2. The parameters of the proposed 2D chaos-based visual clinical EEG signals.

\begin{tabular}{|c|c|}
\hline$F_{C I A X}$ & $\begin{array}{l}\text { chaotic index address assignment process in } \mathrm{x} \text { coordinate } \\
\text { axis }\end{array}$ \\
\hline$C M T_{F X}$ & a chaotic logistic map type of $F_{C I A X}$ \\
\hline$S P_{F X}$ & a initial value of chaotic logistic ma \\
\hline$x_{n}$ & $\begin{array}{l}\text { chaotic sequences which was generated by chaotic logistic } \\
\text { map type } C M T_{F X} \text { and initial value } S P_{F X}\end{array}$ \\
\hline$n_{F X}$ & $\begin{array}{l}\text { security level parameter } \\
\text { discard previous } n_{F X} \text { chaotic sequences }\left(x_{n}\right) \text { to increase } \\
\text { encryption robustness. }\end{array}$ \\
\hline$\delta_{F X}$ & $\begin{array}{l}\text { security level parameter } \\
\text { If } x_{n} \text { is larger than } \delta_{F X} \text {, discard the chaotic point } x_{n} \text { to in- } \\
\text { crease encryption robustness. }\end{array}$ \\
\hline$G_{C C S X}$ & chaotic candidate point generation in $\mathrm{x}$ coordinate axis \\
\hline$C M T_{G X}$ & a cha \\
\hline$S P_{G X}$ & a initi \\
\hline$G_{X}$ & $\begin{array}{l}\text { ated by chaotic logistic } \\
S P_{G X}\end{array}$ \\
\hline$F_{C I A Y}$ & ess in y coordinate \\
\hline$C M T_{F Y}$ & 0 type of $F_{C I A Y}$ \\
\hline$S P_{F Y}$ & a init \\
\hline$y_{n}$ & $\begin{array}{l}\text { ed by chaotic logistic } \\
\text { FY }\end{array}$ \\
\hline$n_{F Y}$ & $\begin{array}{l}\text { security level parameter } \\
\text { discard previous } n_{F Y} \text { chaotic sequences }\left(y_{n}\right) \text { to increase } \\
\text { encryption robustness. }\end{array}$ \\
\hline$\delta_{F Y}$ & $\begin{array}{l}\text { security level parameter } \\
\text { If } y_{n} \text { is larger than } \delta_{F Y} \text {, discard the chaotic point } y_{n} \text { to in- } \\
\text { crease encryption robustness. }\end{array}$ \\
\hline$G_{C C S Y}$ & eration in y coordinate axis \\
\hline$C M T_{G Y}$ & a chaotic logistic $\mathrm{m}$ \\
\hline$S P_{G Y}$ & $\mathrm{a}$ in \\
\hline$G_{Y}$ & $\begin{array}{l}\text { chaotic sequences which was generated by chaotic logistic } \\
\text { map type } C M T_{G Y} \text { and initial value } S P_{G Y}\end{array}$ \\
\hline$L_{F}$ & the number of input clinical EEG samples per channel \\
\hline$N$ & r of input parallel clinical EEG channels \\
\hline CEX & ly encrypted sequence in the $\mathrm{x}$ coordinate \\
\hline$C E Y$ & $\begin{array}{l}\text { 1D chaotically encrypted sequence in the y coordinate } \\
\text { axis }\end{array}$ \\
\hline CEXY & $2 \mathrm{D} \mathrm{cl}$ \\
\hline CEEG1 & $1^{\text {st }} 2 \mathrm{D}$ chaotically encrypted clin \\
\hline SGEEGN & $2^{\text {nd }} 2 \mathrm{D}$ \\
\hline & correlation coefficient \\
\hline
\end{tabular}

EEG signals. Signals mapping of a 2D chaotic scrambler and a permutation scheme are used to obtain clinical EEG information that requires high-level encryption. Simulation results show that when the correct deciphering parameters are inputted, the signals are completely recovered. This $2 \mathrm{D}$ encryption scheme can also be applied to mobile telemedicine systems in which the EEG signals have a transmission BER of $10^{-7}$. However, signal recovery is not achieved if there is an appreciable error in the input parameters, for example, when the 
initial point error is $0.00000001 \%$. The proposed encryption scheme can be used for the encryption of E-health and M-health biomedical signals.

\section{ACKNOWLEDGMENTS}

The authors acknowledge the support of the teacher research project of National Taiwan Ocean University, 99B60201, Center for Marine Bioenvironment and Biotechnology, NSC 93-2218-E-019-024, and the valuable comments of the reviewers.

\section{REFERENCES}

1. Alfaouri, M., Daqrouq, K., Abu-Isbeih, I. N., and Khalaf, E. F., "Quality evaluation of reconstructed biological signals," American Journal of Applied Science, Vol. 5, No. 1, pp. 187-193 (2009).

2. Andrew, T. P. and Kevin, M. S., "Reconstructing the keystrem from a chaotic encryption scheme," IEEE Transactions on Circuits and Systems I: Fundamental Theory and Applications, Vol. 48, No. 5, pp. 624-630 (2001).

3. Curiac, D. I., Dranga, O., Dragan, F., and Banias, O., "Chaos-based cryptography: End of the road?" Proceeding of IEEE The International Conference on Emerging Security Information, Systems, and Technologies, Spain, pp. 71-76 (2007).

4. Dachselt, F. and Schwarz, W., "Chaos and cryptography," IEEE Transactions on Circuits and Systems-I: Fundamental Theory and Applications, Vol. 48, No. 12, pp. 1498-1509 (2001).

5. EEG Database, from http://kdd.ics.uci.edu/database/eeg/eeg.html.

6. Frank, D., Kristina, K., and Wolfgang, S., "Discrete-time chaotic encryption systems-Part III: Cryptographical analysis," IEEE Transactions on Circuits and Systems-I: Fundamental Theory and Applications, Vol. 45, No. 9, pp. 983-988 (1998).

7. Jakimoski, G. and Kocarev, L., "Analysis of some recently proposed chaos-based encryption algorithms," Physics Letters A, Vol. 291, No. 6, pp. 381-384 (2001).

8. Kocarev, L., "Chaos-based cryptography: A brief overview," IEEE Circuits and System Magazine, Vol. 1, No. 3, pp. 6-21 (2001).

9. Li, Y., Liang, L., Su, Z., and Jiang, J., "A new video encryption algorithm for H.264," IEEE International Conference on Information Communications, and Signal Processing, Bangkok, Thailand, pp. 1121-1124 (2005).

10. Lin, C. F. and Chang, K. T., "A power assignment mechanism in Ka band OFDM-based multi-satellites mobile telemedicine," Journal of Medical and Biological Engineering, Vol. 28, No. 1, pp. 17-22 (2008).

11. Lin, C. F., Chang, W. T., Lee, H. W., and Hung, S. I., "Downlink power control in multi-code CDMA mobile medicine system," Medical \& Biological Engineering \& Computing, Vol. 44, No. 5, pp. 437-444 (2006).

12. Lin, C. F., Chang, W. T., and Li, C. Y., "A chaos-based visual encryption mechanism in JPEG2000 medical images," Journal of Medical and Biological Engineering, Vol. 27, No. 3, pp. 144-149 (2007).

13. Lin, C. F., Chen, J. Y., Shiu, R. H., and Chang, S. H., "A Ka band WCDMAbased LEO transport architecture in mobile telemedicine," in: Martinez,
L. and Gomez, C. (Eds.), Telemedicine in the 21st Century, Nova Science Publishers, Inc, USA, pp. 187-201 (2008).

14. Lin, C. F., Chung, C. H., Chen, Z. L., Song, C. J., and Wang, Z. X., “A chaos-based unequal encryption mechanism in wireless telemedicine with error decryption," WSEAS Transactions on Systems, Vol. 7, No. 2, pp. 49-55 (2008).

15. Lin, C. F., Chung, C. H., and Lin, J. H., "A chaos-based visual encryption mechanism for EEG clinical signals," Medical \& Biological Engineering \& Computing, Vol. 47, No. 7, pp. 757-762 (2009).

16. Lin, C. F. and Li, C. Y., "A DS UWB transmission system for wireless telemedicine," WSEAS Transactions on Systems, Vol. 7, No. 7, pp. 578588 (2008)

17. Man, K. P., Wong, K. W., and Man, K. F., "Security enhancement on VoIP using chaotic cryptography," IEEE Conference on Industrial Electronics, Paris, France, pp. 3703-3708 (2006)

18. Marco, G., Kristina, K., and Wolfgang, S., "Discrete-time chaotic encryption systems-Part I: Statistical design approach," IEEE Transactions on Circuits and Systems-I: Fundamental Theory and Applications, Vol. 44, No. 10, pp. 963-970 (1997)

19. Matthews, R., "On the derivation of a chaotic encryption algorithm," Cryptologia, Vol. 8, No. 1, pp. 29-41 (1989).

20. Murali, K., Yu, H., Varadan, V., and Leung, H., "Secure communication using a chaos based signal encryption scheme," IEEE Transactions on Consumer Electronics, Vol. 47, No. 4, pp. $709-714$ (2001).

21. Naoki, M., Goce, J., Kazuyuki, A., and Ljupco, K., "Chaotic block ciphers: from theory to practical algorithms," IEEE Transactions on Circuits and Systems-I: Fundamental Theory and Applications, Vol. 53, No. 6, pp. 1341-1352 (2006).

22. Naor, M. and Shamir, A., "Visual cryptography," Advances in Cryptography Eurocrypt Conference, Perugia, Italy, pp. 1-12 (1994).

23. Ou, C. M., "Design of block ciphers by simple chaotic functions," IEEE Computational Intelligence Magazine, Vol. 3, No. 2, pp. 54-59 (2009).

24. Pareek, N., Patidar, V., and Sud, K., "Discrete chaotic cryptography using external key," Physics Letter A, Vol. 309, No. 1, pp. 75-82 (2003).

25. Ranjan, B. and Saumitr, P., "Novel compression and encryption scheme using variable model arithmetic coding and coupled chaotic system," IEEE Transactions on Circuits and Systems-I: Fundamental Theory and Applications, Vol. 53, No. 4, pp. 848-857 (2006).

26. Sobhy, M. I. and Shehata, A. E. D., "Secure e-mail and databases using chaotic encryption," Electronics Letters, Vol. 36, No. 10, pp. 875-876 (2000).

27. Wheeler, D. D., "Problems with chaotic cryptosystems," Cryptologia, Vol. 13, No. 3, pp. 243-250 (1989).

28. Yan, M., Bourbakis, N., and Li, S., "Data, image, video encryption," IEEE Potentials, Vol. 23, No. 3, pp. 28-34 (2004).

29. Yang, T., Wu, C. W., and Chua, L. O., "Cryptography based on chaotic systems," IEEE Transactions on Circuits and Systems-I: Fundamental Theory and Applications, Vol. 44, No. 5, pp. 268-271 (1997).

30. Yen, J. C. and Guo, J. I., "Efficient hierarchical chaotic image encryption algorithm and its VLSI realisation," IEE Proceedings Vision, Image, and Signal Processing, Vol. 147, No. 2, pp. 167-175 (2000).

31. Zhou, H. and Ling, X. T., "Problems with the chaotic inverse system encryption approach," IEEE Transactions on Circuits and Systems-I: Fundamental Theory and Applications, Vol. 44, No. 3, pp. 268-271 (1997). 